\title{
Residual Elastic Stress in Historical Coins as a Criterion for Authentication
}

\author{
Alexander Joseph Kossolapov", Ksenia Sergeevna Chugunova \\ Department for Scientific Examination, Authentication of Works of Art, State Hermitage Museum, Saint Petersburg, Russian Federation \\ Email address: \\ expert@hermitage.ru(A.J. Kossolapov), askachu@yandex.ru (K. S. Chugunova) \\ ${ }^{*}$ Corresponding author \\ To cite this article: \\ Alexander Joseph Kossolapov, Ksenia Sergeevna Chugunova. Residual Elastic Stress in Historical Coins as a Criterion for Authentication. \\ Science Journal of Chemistry. Vol. 9, No. 5, 2021, pp. 121-128. doi: 10.11648/j.sjc.20210905.12
}

Received: August 20, 2021; Accepted: September 4, 2021; Published: September 10, 2021

\begin{abstract}
Authentication of coins is frequently presenting complicated problem in numismatics practice. Metal composition only may not be enough to identify forgeries, notably when one deals with noble metals - silver, gold or electron. Isotopic ratios, providing in certain cases information regarding metallic ore source (geographical provenance), require a sample to be extracted for MS-analysis while sampling is not permissible for numismatic material in principle. To solve the problem it becomes crucial, in addition to metal's composition, to establish the method used for a coin's manufacturing, as forgers are rarely realizing or following the method that was used in the original historical production. Conventional visual studying under microscope is not always being enough to understand how the coin was produced while metallographic examination of polished and etched metal samples, which might provide necessary information, is not applicable here for its destructive character. Necessary information regarding manufacturing could have been extracted from residual stress analysis, but, in general, there were very few published works regarding this kind of analysis for coins, and those few did not consider any connection between manufacturing process and residual stress in metal. The expected types of residual elastic stress arisen under historically known methods of coins production (casting in hot or cold mold, striking hot or cold metal) are considered in this work. On this base, non-destructive X-rays diffraction method $\left(\operatorname{Sin}^{2} \Psi\right.$-method) is offered to distinguish between various methods of manufacturing. The results may be applied in museum's laboratory as useful criterion for authentication of coins and medals.
\end{abstract}

Keywords: Numismatics, Authentication, Cast Coins, Struck Coins, Residual Stress, XRD, Method of Production

\section{Introduction}

In many cases of numismatic examination of coins it is difficult to determine the method of their production despite the use of a magnifying glass or binocular microscope. In particular, it is not always possible to detect a sprue (casting gate) or gas pores on a cast coin or to detect traces of punching by examining surface relief on stricken coins of archaeological provenance.

It is not possible on practice to extract a sample from a coin for establishing manufacturing method by means of conventional metallographic microscopic examination. In experiments of N. Pistofidis et al [1], that approach was chosen for Greek coins of relatively low value and for scientific purposes only.

In this work, non-destructive X-Ray diffraction analysis
(XRD) is proposed as a tool to establish the production method. The type of residual elastic stress preserved in the metal is chosen as a reliable criterion.

Residual stress in coins/medals did not attract much attention from scientists until now and very few works related to this question have been published. In early work [2] by this author it was established that many cast coins demonstrate dots (or blocks) on diffraction lines, the effect being a result of the formation of large crystallites on a surface under slow cooling. This effect may be absent in cases when metal cools rapidly or when cold casting molds are used ("chill casting"). For this reason, it is not always possible to detect cast coins solely on the presence or absence of dots on diffraction lines.

R. Yokoyama and Y. Takahashi [3] examined residual stress in an original "Japanese" coin manufactured in China 
under the Northern Song Dynasty and in its Japanese regional imitation. The authors' conclusions were based only upon differences in the residual stress values measured by XRD methods for the original coin and the forgery, but other factors may be involved. Such a difference is not necessarily connected with the coin's authenticity (as both coins were probably cast in similar cold molds) but may result from many other factors including mold thickness, thermal conductivity and metal composition.

In a methodological publication of the Rigaku Residual Stress Analysis Group, Japan [4], compressive stress was measured in a 10 Yen Japanese coin merely as an example of the XRD application without any specific conclusions.

Among non-X-ray methods, the works of Yanxia Xie et al. [5] are worth mentioning. The authors used time-of-flight neutron diffraction (TOF-ND) to reveal textures in an authentic ancient Greek coin and its imitation. No attention was paid to residual stress. The authors were based at Los Alamos National Laboratory and their approach required instrumentation available only in highly specialized neutron research centers. They managed to reveal "very weak" textures in both authentic and fake Greek coins that probably indicated that the temperatures during the manufacturing process for both coins were high enough to permit preferential orientation of the metal crystals. One would doubt that the fake coin was manufactured by hot-striking that was specific for authentic coins of that type. Since no difference was revealed in the metal compositions of the tested coins either, their work did not identify any technical difference between fake and original.

In the work [6] it has been tried for the first time to connect the residual elastic stress in coins with the method of manufacturing. It was due to useful remarks of our reviewers that we considered to specify in this paper the connection between historically known methods of coins manufacturing processes and formation and type of residual stress. The results of this study may help numismatists in complicated cases of coins authentication.

\section{XRD Application for Coins}

\subsection{Residual Elastic Stress and Method of Coins Manufacturing}

XRD is based on the registration and measurement of the spatial distribution and intensity variations produced by interference effects on monochromatic coherent X-rays scattered by a crystalline lattice. Almost all metals are polycrystalline substances and, because of this, XRD presents a powerful method for their examination. The interference pattern is frequently, though imprecisely, called a diffraction pattern.

When monochromatic radiation with a wavelength comparable to the interatomic distances of a crystal hits the material this radiation is scattered only in specific directions. These directions are determined by parameters of the crystalline lattice and the radiation wavelength.
If the incident radiation falls on a surface of a polycrystalline substance at angle $\theta$, the diffracted radiation may be "reflected" from a certain domain of parallel crystalline planes at the same angle $\theta$. However, in contrast to mirror-like reflection, such reflection takes place only for definite discrete values of $\theta$, which are determined by Bragg's law [7]:

$$
2 \mathrm{~d} \sin \theta=\lambda \mathrm{k}
$$

where $\mathrm{d}$ - interplanar distance for a given group of "reflecting" parallel crystalline planes in crystal, $\lambda$ wavelength of incident irradiation, $\theta-$ an angle between direction of incident irradiation and "reflecting" planes, $\mathrm{k}-$ an integer, defining the so-called "order" of diffraction (normally $\mathrm{k}$ is taken 1 , as reflections for higher orders have much smaller intensities), see Figure 1.

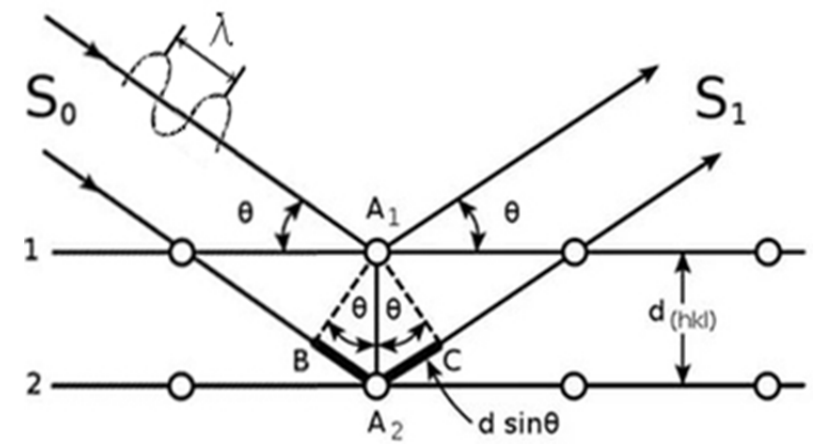

Figure 1. Diffraction of monochromatic X-rays on crystalline lattice.

If the set of all diffraction angles $\left\{\theta_{\mathrm{n}}\right\}$ is measured, all $\left\{\mathrm{d}_{\mathrm{n}}\right\}$ values can be calculated using (1). The $\left\{d_{n}\right\}$ set uniquely defines the given crystal. Tabulated sets in available databases permit a given substance to be uniquely identified, providing the basis for "X-ray phase identification".

All metals used for coin production are polycrystalline substances, i.e. they consist of many randomly oriented small monocrystals. A polycrystalline substance, as the Bragg's law predicts, scatters the incident parallel X-Ray beam into a set of cones with cone angles $2 \theta_{\mathrm{n}}$, Figure 2 .

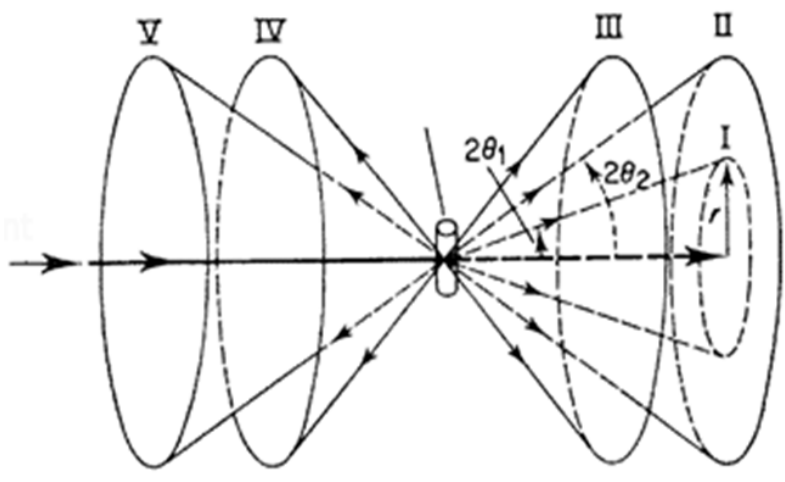

Figure 2. Diffraction cones.

Deviations of interplanar d-distances from tabulated values may arise due to two main reasons:

"Elastic stress" of the first type manifests itself as a 
systematic shift in values of measured diffraction angles $\theta_{\mathrm{n}}$ in the same direction (all $\theta_{\mathrm{n}}$ are larger or smaller than corresponding tabulated values). All resulting $\mathrm{d}_{\mathrm{n}}$, calculated from these values, are correspondingly smaller or larger than tabulated [8] values for a given stress-free material, see Figure 3.

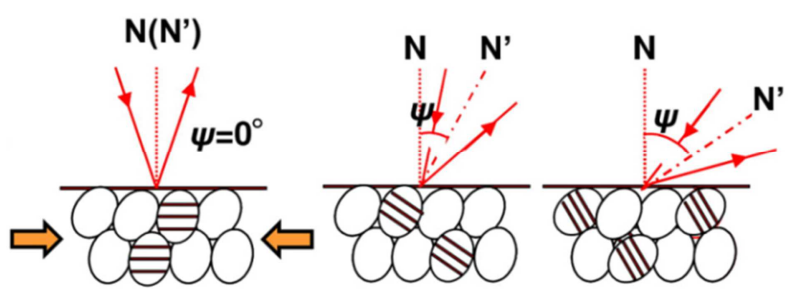

Figure 3. Red arrows indicate directions of incident and diffracted X-rays. An angle between these arrows in this notation is $(\pi-2 \theta)$. Stress directed along yellow arrows makes interplanar distances $d_{n}$ bigger for planes oriented in parallel with the stress direction and smaller for the planes oriented at angle to it. $\Psi$ - the angle between normal to coin's surface and normal to diffracting planes.

Stress of the second type manifests itself as widening of diffraction lines due to arbitrarily changed distances $\left(d_{n}\right.$ $\pm \Delta d_{n}$ ) depending upon the stress distribution inside the metal and crystals' orientations. In contrast to stress of the first type, average d-distances for this case are equal to corresponding tabulated values for stress-free substance, but diffraction lines are wider than it would be expected.

The second type stress is not the only reason for diffraction lines widening. The line width is also dependent upon the diffracting crystals size. From general theory it follows that, within certain size limits, the smaller the crystals become, the wider the lines become, and vice-versa.

The reason for this widening of diffraction lines may be established based upon a different angular dependence of the line width. A stress-induced increase of line width changes as $\tan (\theta)$, while that dependent upon the crystal size changes as $\cos ^{-1}(\theta)$. [9]

In this work we consider the first type of stress only, as the second type may appear not only as a result of mechanical deformation in metal during minting but also as a result of crystal growth during cooling of cast coins. Thus it does not seem reasonable to distinguish cast coins from the stricken ones based on diffraction line widths.

At the same time the first type stress measurement permits the distinction to be made, as the stricken coins demonstrate compressive stress on their surface $(-\sigma)$, while cast coins demonstrate various types of stresses including no stress, compressive stress or, under specific conditions of cooling, tensile stress $(+\sigma)$ (see 3 below).

It must be noted that the stress that can be measured with $X$-ray diffraction is directly connected with crystal lattice deformation (interatomic distance changes) and, because of that, it is elastic by nature. The presence of non-elastic (plastic) deformation in metal (i.e. the material does not return to its original shape after the initial stimulus-load is removed) is not directly connected with the existence of the first type of elastic stress in coins. Residual elastic stress may arise in material subjected to plastic deformation only when the plastic deformation through the sample volume is not uniform. In the stamping process a stress greater than the elasticity limit for a given metal produces both types of deformations - elastic and non-elastic/plastic. Elastic stress disappears after the strike is finished, non-elastic stress remains. After flattening, the blank (flan) becomes thinner and wider and it retains the image engraved in the punch. If the plastic deformation is not uniform in its volume, residual elastic stress will be present in the metal.

Intuitively it may seem from this that a relationship between visible plastic deformation and residual elastic stress may not work as it does in reality. It would seem, for instance, that interplanar distances are smaller for planes oriented in parallel with the coin surface subjected to punching in the directed normal to the surface, i.e. that interplanar distances follow the metal's flattening. This idea is totally wrong. In reality the strike on the metal results in non-uniform plastic deformation under which metal layers situated closer to the surface are plastically stretched more than the layers lying deeper in the volume. The resulting effect is an elastic compression in the surface layer in a direction parallel to the surface - the distances between crystalline planes parallel to the surface become larger (not smaller, as it would seem) than for stress-free metal. In metallography such an effect is known as cold hardening. Compressive residual stress of the first type is present in all stricken coins, no matter if the coins were stricken cold or hot. This conclusion goes well in line with experiments of Japanese authors who found (using Rigaku Auto-Mate 2 Xray diffractometer) the presence of residual compressive stress in forged Ag-Au Koban-type coins, while the stress value was greater on the stamped areas of the surface than on its flat areas [10]. Indeed, local stamping of marks on previously forged flat plates of Koban-type coins might add additional residual stress on those marks compared with unstamped area.

The difference between cold and hot stricken coins is the temperature of the flan under striking - for the latter it is kept above the temperature of recrystallization for a given metal, while for the former the temperature is at ambient, that is, well below its recrystallization point. During hot-striking, the pressure required for metal deformation becomes much smaller (for increased plasticity and the practical absence of elasticity) permitting coins of greater size with a deeper profile to be struck using the hand-hammering specific for ancient times.

While hot-punching permits the manufacture of large coins with deep relief by hand-hammering, the surface of such coins is not as smooth as those of the cold-striking process. Another common feature for hot-stricken coins met frequently is a compositional difference. Many makers added up to $10-12 \%$ of lead into silver (or copper) with the probable goal to increase the metal's plasticity at a temperature below the recrystallization point for pure silver. (See the case below of an antique Greek tetradrachm that contains $\sim 10 \%$ of lead.).

For coins cast into a cold mold the surface cools faster than its inner part. A temperature gradient directed from the 
coin's surface to its center arises and, as a consequence, different thermal expansion of inner and outer layers takes place. The surface layer may contract in size more than the hotter inner core may permit. As the result, the surface layer experiences greater plastic deformation (stretching) compared to the neighboring undersurface layer of metal. A difference in plastic deformation remains upon cooling resulting in formation of compressive residual elastic stress on the coin's surface in the direction parallel to that surface (and corresponding sub-surface residual tension). The presence of residual compressive stress for cast medals was previously reported in [11].

Coins cast into a cold mold commonly demonstrate quite a porous surface (so-called "air-pockets") clearly seen by the naked eye. The presence of deep pores on a coin's surface alone is enough to suspect cold casting.

The situation becomes different for coins cast in a hot mold. The mold may in this case be heated above the melting point of the metal to provide easy flow of the liquid metal into the mold. A very hot mold, for instance, may be absolutely necessary if a multi-faceted mold is used to cast several copies simultaneously.

As the thermal conductivity of mold material (typically a fired clay+sand composition) is several dozen times smaller than the thermal conductivity of any metal, no noticeable temperature gradient arises in the metal during the cooling and, as the result, residual stress is absent or negligent. Due to a longer period of hardening big crystallites grow, resulting in disruption of the X-ray diffraction lines with big dots (so-called "blocks"). The normally linear graph 20$\sin ^{2} \Psi$, used to calculate the value of residual first type stress (see below), demonstrates strong deflection from linearity that gives a large error in the calculated value of the stress. As the stress value in this case is small or negligent, the error value happens to be several times greater than the stress value.

It must be emphasized that when a mold is heated above the metal's melting point and cools very slowly the thermal flux may propagate sometimes in a direction from the mold internal wall through the metal to the outside. (For instance, the thermal flux can exit the mold through a large sprue to atmosphere.) In this case, the metal's surface contacting the hot mold can be hotter than the internal part of the casting as a consequence of the insulating effect of the heated mold.
This situation is opposite to the case of casting into the cold mold and, consequently, the residual surface stress becomes tensile (not compressive as it is for casting in cold mold!).

The surface of a coin cast into a hot mold looks smoother than it does for a coin cast into cold mold. The appearance of coins cast into a hot mold may be much like the appearance of stricken coins. If a sprue attachment is thoroughly erased by polishing, the coin cast into hot mold can be easily mistaken for a struck coin. However in this case, the XRD residual stress analysis provides the necessary information for distinguishing the two by the following criteria:

1. Residual stress for stricken coins is compressive while for coins cast into a hot mold, the residual stress is negligible or sometimes tensile rather than compressive;

2. Under very slow cooling, large crystals (larger than 100 $\mathrm{nm})$ grow in the metal, resulting in large elongated dots (blocks) on the diffraction lines, see Figure 4. The blocks prevent a reliable measurement of diffraction angles that makes it difficult to measure residual stress with any precision. But the presence of blocks alone is sufficient to identify coins cast into hot mold.

Under punching, the larger crystallites in cast blanks fragment. Consequently the blocks on diffraction lines are absent for any kind of stricken coin [6].

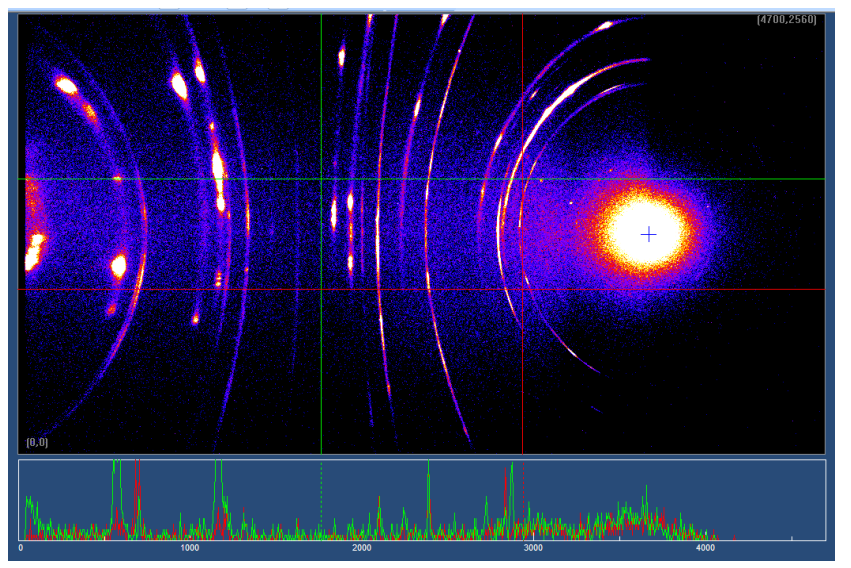

Figure 4. Blocks on diffraction lines for cast coin

Predicted features for coins manufactured by various methods are summarized in Table 1.

Table 1. Expected features for stricken and cast coins.

\begin{tabular}{llll}
\hline Type & Surface & Blocks on diffraction lines & Residual elastic stress on the surface \\
\hline Stricken hot & Flat, no pores & Absent & Compression usually exceeding 200 MPa \\
Stricken cold & Flat, no pores & Absent & $\begin{array}{l}\text { Compression usually below 140 MPa for silver and copper based } \\
\text { coins; smooth surface }\end{array}$ \\
$\begin{array}{ll}\text { Cast, cold mold } \\
\text { Cast, hot mold }\end{array}$ & Pores, shells, air pockets & Absent & Compression, pores on the surface \\
\hline
\end{tabular}

\subsection{Theory of the XRD $\sin ^{2} \Psi$-Method for Residual First Type Elastic Stress Measurement}

XRD provides a convenient non-destructive method to measure the value of surface parallel first type elastic stress and its sign, the latter indicating compression by (-) or tension by $(+)$. The so-called " $\sin ^{2} \Psi$-method" is routinely used for this purpose. The method is based on predictable dependence of a chosen diffraction angle $2 \theta$ on the angle $\Psi$ 
between the normal to the coin surface $(\mathrm{N})$ and the normal to the diffracting crystalline planes (N'), see Figure 3. A good practical description of the " $\sin ^{2} \Psi$-method" may be found in [12].

The theory predicts that dependence of diffraction angle $2 \theta$ on $\sin ^{2} \Psi$ should be linear, i.e. the value $\Delta 2 \theta / \Delta\left(\sin ^{2} \Psi\right)$ is constant for the given material and the corresponding value of first type residual elastic stress $\sigma$ may be found from expression:

$$
\sigma=\mathrm{K} \cdot \frac{\Delta 2 \theta}{\Delta\left(\sin ^{2} \Psi\right)}
$$

where $\mathrm{K}$ is so-called stress constant

$$
\mathrm{K}=\frac{-E}{2(1+v)} \cot \left(\theta_{\mathrm{o}}\right)\left(\frac{\pi}{180}\right)
$$

E- Young modulus, $v$ - Poisson ratio, $\theta_{0}-1 / 2$ of the diffraction angle for stress-free material as tabulated.

The value $\Delta 2 \theta / \Delta\left(\sin ^{2} \Psi\right)$ may be found as a slope of $2 \theta$ $\sin ^{2} \Psi$ plot, the latter, as it is said above, is a straight line.

If this slope is positive $\left(\Delta 2 \theta / \Delta\left(\sin ^{2} \Psi\right)>0\right)$, the stress $\sigma$ will be negative, indicating compressive stress and, if the slope is negative $\left(\Delta 2 \theta / \Delta\left(\sin ^{2} \Psi\right)<0\right)$, the stress $\sigma$ is positive, indicating tensile stress on the surface of a coin.

\section{Experiment}

Residual elastic stress of the first type in coins has been measured in our experiments using a Rigaku (Japan) X-ray diffractometer D/MAX RAPID II with automated stressmeasuring stage. Measuring parameters in our experiments were: generator: $40 \mathrm{kV}$, current: $30 \mathrm{~mA}$, anode: $\mathrm{Cu}$, graphite monochromator, collimators: $0.8 \mathrm{~mm}, 0.3 \mathrm{~mm}$, acquisition: 60 s for each $\Psi$ value.

Rigaku software 2DP v.2.4.2 was used in experiments for minted and cast coins of different ages and centers of production. The Young's modulus was taken as $80 \mathrm{GPa}$ for silver-based coins and $110 \mathrm{GPa}$ for copper-based ones. The Poisson ratios were 0.370 and 0.330 for silver and copper, respectively. The measurement diffraction angle was $2 \theta_{0}=$ 134.955 for silver and 135.60 for copper.

The experimental installation and results are shown in Figures 5a-d.

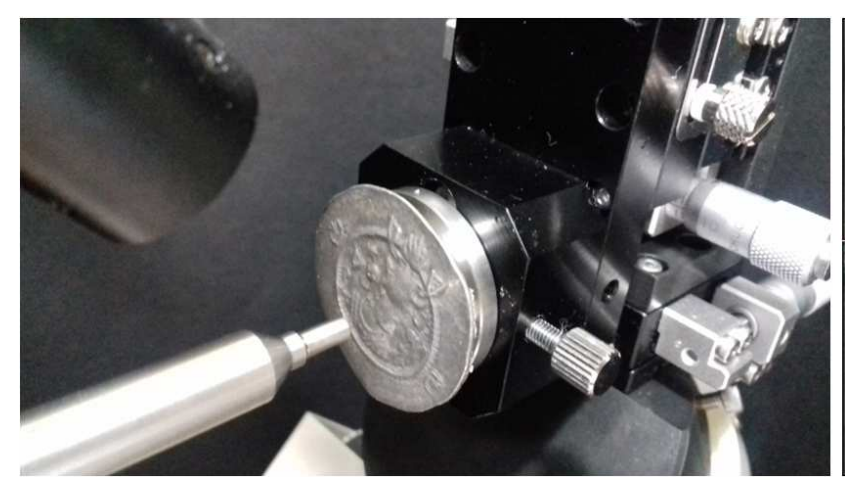

A

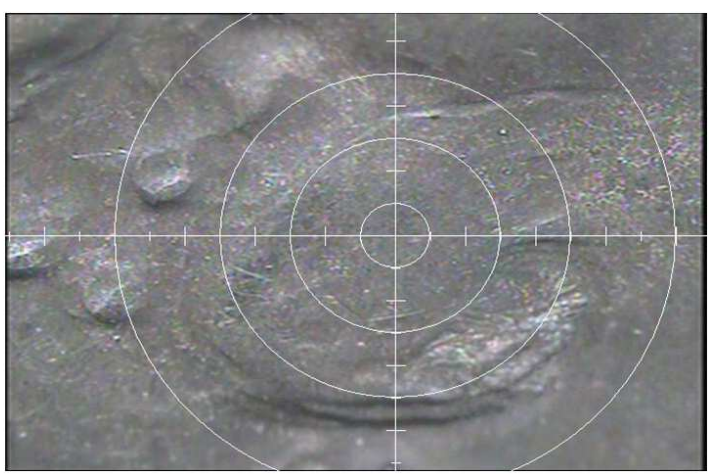

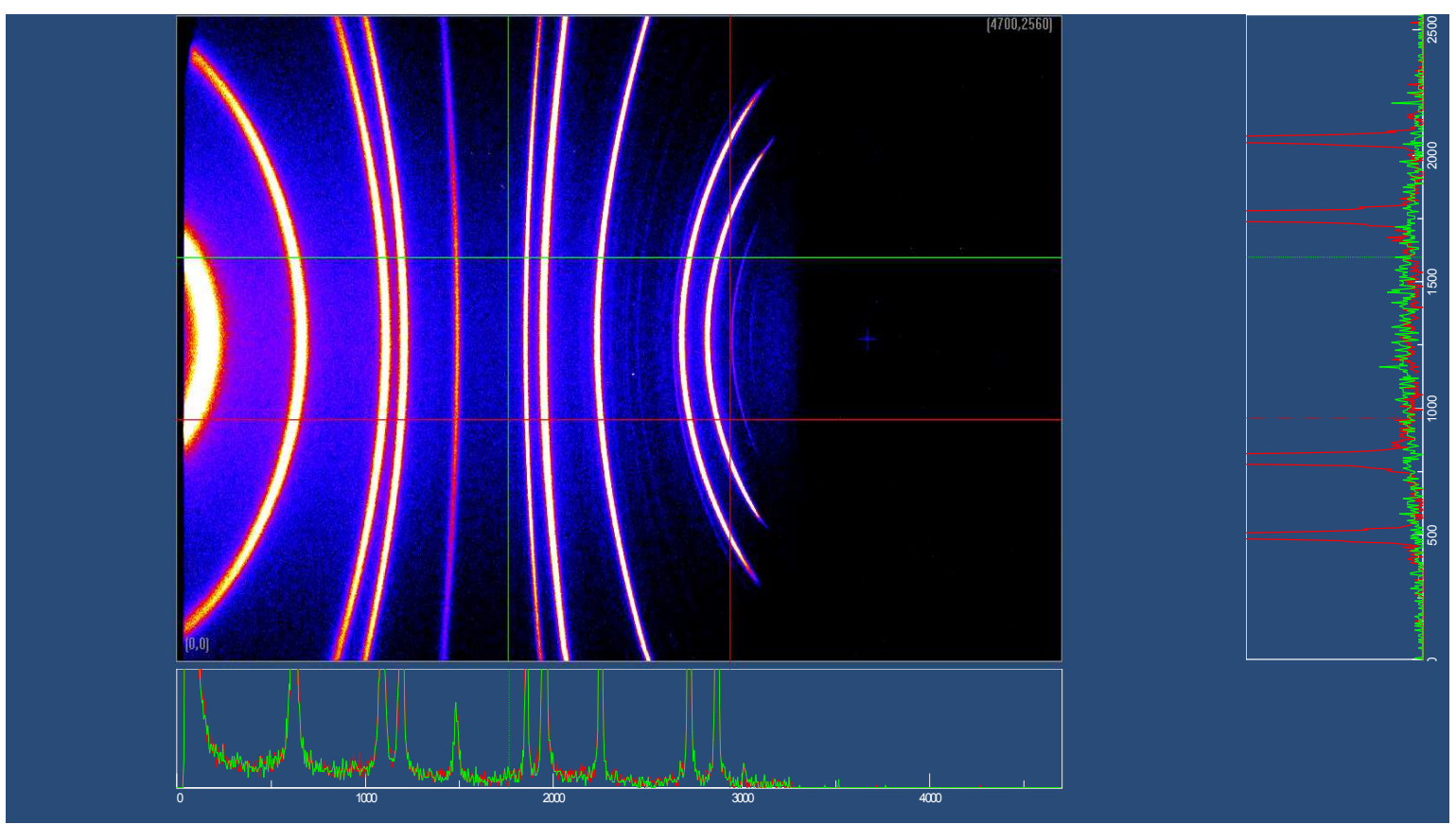




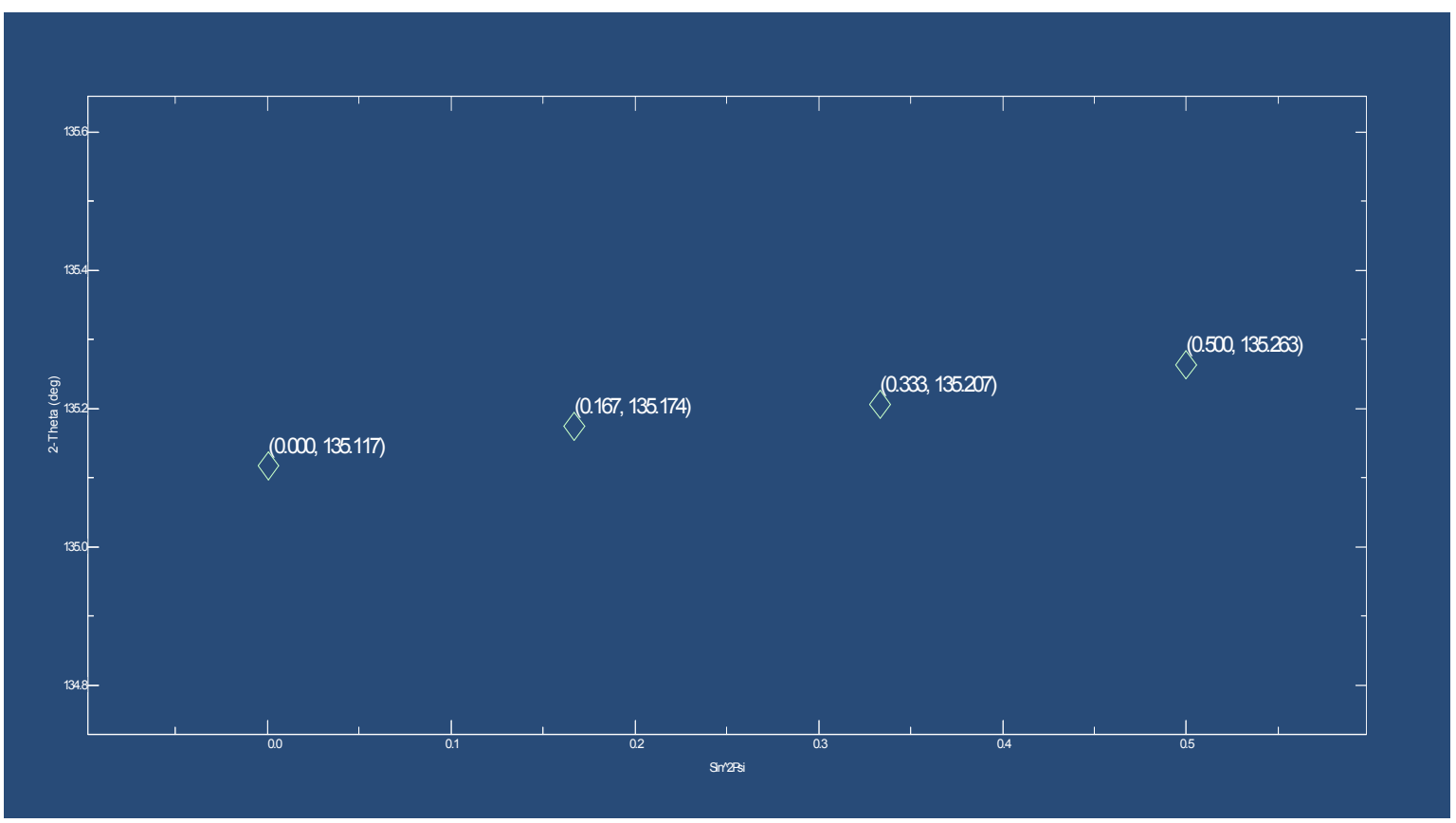

$\mathrm{D}$

Figure 5. Silver drachm, Iran, Sasanides, Kavad 1 (488-531 A.D.). 4.188 g.

A- the drachm on the diffractometer stage; B- surface spot selection using telescopic digital camera; C- X-ray diffractogram; D- $2 \theta$ - $\sin ^{2} \Psi$ graph, positive slope indicates compressive stress.

Material - Ag, diffraction angle $2 \theta_{0}=134,955^{\circ}$, wavelength $\lambda=1,540059(\AA)$,

Young's module E=80 (GPa), Poisson ratio v=0.370, Stress constant $\mathrm{K}=-211.312$ (MPa/deg., Residual stress measured $\sigma=-(59.801 \pm 4.239)$ MPa, compression.

This coin has rather flat surface and it might have been cold-struck.

Table 2 gives some experimental results for various coins, including the measured residual stress and conclusion regarding a method of production for the given coin.

Table 2. Experimental results for various coins produced by various methods.

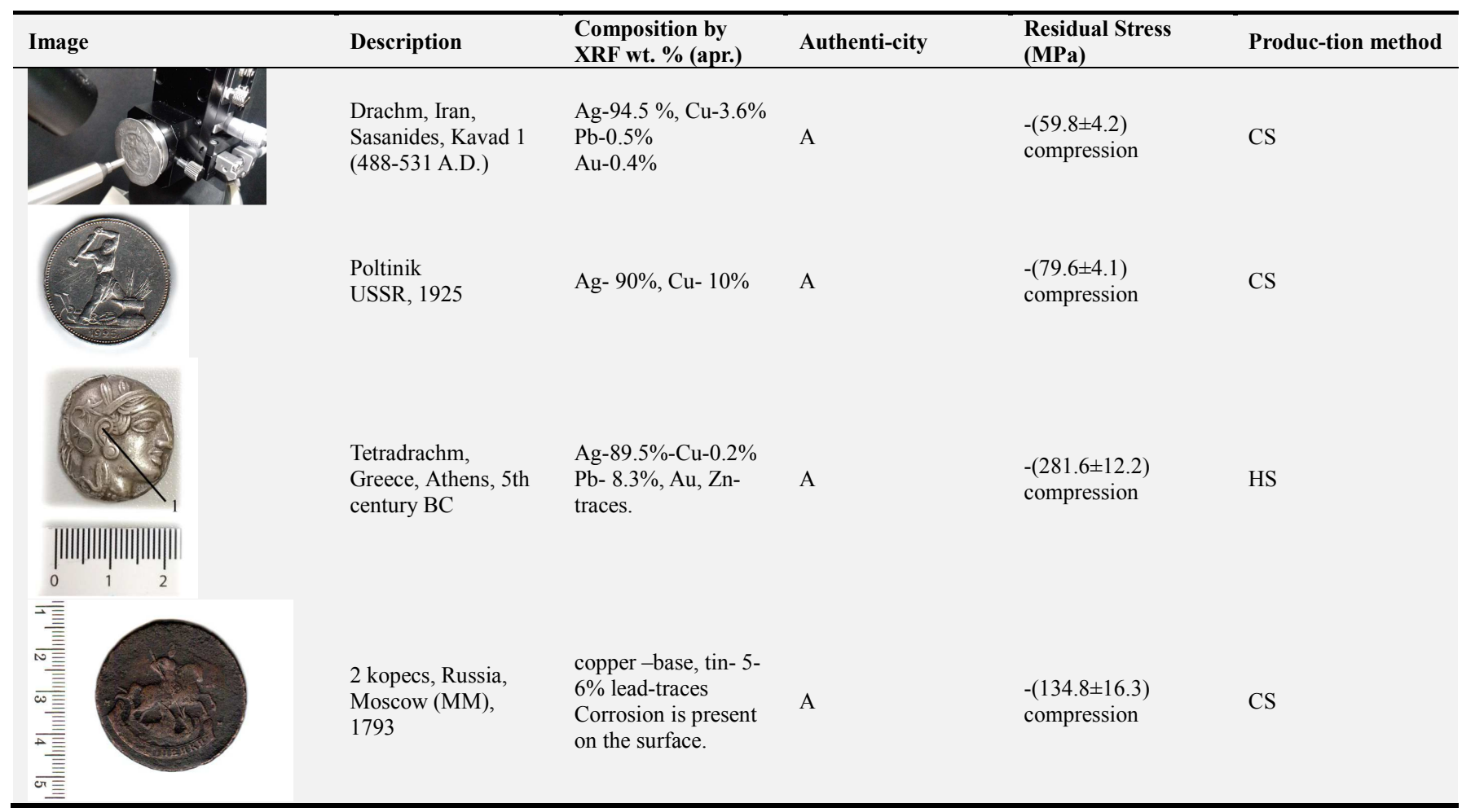




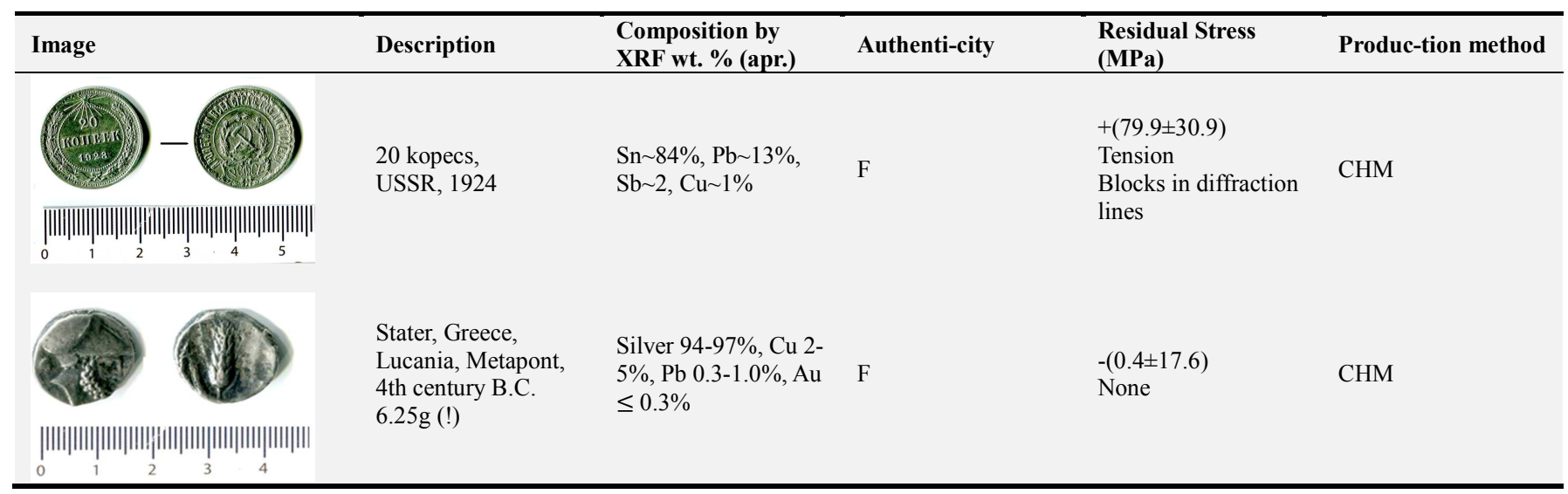

A-authentic, F-faked coin, CS-cold-stricken, HS-hot-stricken, CHM-cast in hot mold.

\section{Surface Corrosion and Residual Elastic Stress}

In coins of both types - stricken or cold-cast, the plastic stretching gradient persists and the plastic deformation of the given layer of metal is depth-dependent.

The main origin of residual elastic stress in struck coins is that the stamped surface area, bearing a three -dimensional image profile, becomes much larger than the surface area of the flan prior to punching. For this reason, the surface layer is plastically stretched. The stretching is present under the surface as well, but its value falls with depth. This stretching gradient is the reason why residual elastic compression is present on the surface. For a balance of stresses to exist, the sub-surface must demonstrate a tensile stress equal in absolute value to the surface compression. If one hypothetically were to split the metal by depth into thin, incremental layers, each sublayer may be understood to be subject to two stresses - tension from the neighboring layer above and compression from its neighboring sublayer. The resulting stress in each increment will be tensile because tension from above is greater than compression from below (the stretching gradient falls with depth).

When corrosion removes metal from the surface in which the stresses are preserved, by their conversion to corrosion products, the surface sublayer of metal becomes the new surface layer. It is no longer under tensile stress from the missing upper layer and its residual elastic stress becomes compressive.

For struck coins the stretching gradient may become very small at a depth exceeding the coin's profile (image) height. For this reason, very small or no residual stress can be measured when the profile metal turns completely into corrosion products.

In coins cast into a cold mold the surface cools faster than the interior and the surface layer volume becomes smaller than it is necessary to fit the hotter internal part. Thus the surface layer is plastically stretched more than its sublayer and the coin demonstrates residual elastic compressive stress. The stretching gradient, due in this case to the high thermal conductivity of metals, may extend throughout the whole coin's volume, as the center of the core remains the hottest point until the casting cools to ambient temperature. Consequently the residual stress remains to be measured in coins cast into a cold mold, no matter how deep the extent of corrosion might be.

Hot-struck coins, where the stretching gradient is formed due to both effects - striking and fast cooling, should demonstrate corrosion-dependable features common for both types of coins - the struck ones and the ones cast into cold mold.

By this line of reasoning, coins cast into a hot mold (which normally demonstrate an absence of elastic residual stress) are not susceptible to the loss of their stress characteristics.

It should be emphasized that removing corrosion products from newly-excavated archaeological coins by mechanical cleaning such as very rough polishing, sand-blasting or laser cleaning (perhaps with the exception of newer femtosecond lasers) leads to an increase of residual elastic compression. From this point of view, chemical cleaning or femtosecond laser conservation treatment are recommended to preserve the character of residual stress. In many cases, when corrosion on the higher parts of a coin's relief is thin enough for diffraction lines of the underlying metal to be detected, the measurement can be done prior to cleaning or conservation.

\section{Conclusion}

1. The XRD residual stress examination provides a criterion to establish the manufacturing method for coins. In particular, all types of stricken coins show residual compression on their surface while hot-stricken coins show much bigger residual stress in comparison with cold-stricken coins.

For cast coins the XRD characteristics of residual stress plus blocks on diffraction lines, in combination with the surface appearance and sharpness of image details, permit a distinction to be made between castings into cold or hot molds. This criterion has the potential to become universal in numismatics, provided the necessary assistance from a laboratory is available. 
2. The following points should be kept in mind:

a. Casting and stamping processes may have other distinctive features in addition to residual stress and surface appearance. For instance, in our work we encountered several types of German thalers of the 16th century, probably manufactured by hot-striking with mechanical press. The thalers demonstrated texture - a preferred direction in orientation of the crystallites. The presence of texture results in violation of linearity in the $2 \theta-\sin ^{2} \Psi$ plot and greater errors in measured values of residual stress should be expected.

b. Some other methods of casting are left outside the scope of our current research. In this work we address casting in either a hot or cold mold. We have not encountered any coins known with certainty to have been manufactured by any type of die-casting. Neither can we try to predict the kind of residual stress exhibited by this type of coin (if such coins have been ever produced by fakers).

Under injection molding or mechanical treatment (grinding, polishing etc.) of a cast coin's surface, residual stress may show patterns different from the ones we described here. For example, after extensive polishing or sand -blasting a hot-cast coin can show residual compression inherent for cold or even hot striking.

c. The question of relaxation time for stresses is not examined here. It is conceivable that study of stress relaxation effects could lead to semi-quantitative dating of coins based on instrumental analysis. This task needs experimental study of the dependence of stress relaxation upon annealing time and temperature, with extrapolation to average room temperature.

\section{Acknowledgements}

The authors greatly appreciate generous assistance of $\mathrm{Mr}$. John Twilley (NY, USA) who made useful remarks and who helped us in English editing of the text.

\section{References}

[1] N. Pistofidis et al. "An Estimate of the Minting Method of Three Silver Coins of the $3^{\text {rd }}$ Century B.C. Through their Microscopical Study" Physica B (2010), DOI: 10.1016/j.physb.2010.01.129.

[2] A. Kossolapov A., L. Viazmenskaya "Application of X-ray Diffraction Analysis for the Identification of Manufacturing Method for Silver-made Museum Objects", Soobschenia Gosudarstvennogo Ermitajha, 1974, XXXIX, 68-70 (in Russian, English abstract).

[3] Ryouichi Yokoyama, Yoshihiro Takahashi Investigation for the Forgery of the Old Coin with X-Ray Residual Stress Measurement. Journ. Soc. Mat. Sci. Japan, v. 65, No 12, pp. 877-882, Dec. 2016.
[4] Rigaku Residual Stress Analysis Group. https://alexzevalkink.files.wordpress.com/2017/04/gens_khp2 _00009_eng1.pdf

[5] Y. Xie, L. Lutterotti, H.-R. Wenk, and F. Kovacs, "Texture analysis of ancient coins with TOF neutron diffraction", Journ. Materials Science, 39 (10): 3329-3337, 2004.

[6] Kossolapov A. J., Chugunova K. S. "Residual Stress in Struck and Cast Coins", Insight- Non Destructive Testing and Condition Monitoring", V. 62, No 3, 139-144, March 2020.

[7] Harold P. Klug, Leroy. E. Alexander "X-Ray Diffraction procedures for Polycrystalliine and Amorphous Materials", John Willey \& Sons, NY, 1974, pp. 960.

[8] Internationa 1Centre for Diffraction Data (ICCD) PDF-4+ 2021/

[9] Scott A. Speakman "Estimating Crystalline Size Using XRD" http://prism.mit.edu/xray/oldsite/crystalsizeanalysis.pdf

[10] Kirino Famiyoshi et al. "Researching of Producing Techniques of Koban and Ginban manufacturing in Edo Era" (in Jaanese. with English summary), Journ. Japan. Inst. Metals@Materials, 2019, 83 (5), 148-156.

[11] Kirino Famiyoshi et al." Production Techniques of Coin-type Medal Rewarding Contributions Manufacturing in the Momoyama-era"', Journ. Japan. Inst. Metals@Materials, 2017, 81 (3), 101-108.

[12] M. E. Fitzpatrick, A. T. Fry et al. "Determination of Residual Stresses by X-ray Diffraction (Issue 2)" https://www.npl.co.uk/gpgs/residual-stresses-xray-diffraction

\section{Biography}

Alexander Joseph Kossolapov Ph.D. (1980, Optics), Head of Department for Scientific Examination/Authentication of Works of Art of the State Hermitage Museum, St. Petersburg, Russia. He has been working in the Hermitage Museum since 1972. In 1990-1995 worked in the Getty Conservation Institute in LA and in Los Angeles County Museum of Art (USA). Numerous publications (including monograph and manual for University students) in the field of IRR and X-Radiography theory and practice, in scientific examination and authentication of paintings, drawings, jewelry, coins/medals and other objects of art and archaeology. Leader of the project "Dating Gold Using He-U Radiogenic Clock".

Mailing: The State Hermitage Museum, 2, Dvortsovaya square, Saint Petersburg, Russia 190000.

Ksenia Sergeevna Chugunova, MS (1996, Chemical Technology of Materials \& Electronics), MS (2006, Archaeology). Senior Scientist, Department for Scientific Examination/Authentication of Works of Art, The State Hermitage Museum. She has been working in the Hermitage Museum since 1999 specializing in SEM, XRD, XRF. Author and co-author of numerous papers on the topic of archaeological metals and ceramics research. Took part in the project "Dating Gold Using He-U Radiogenic Clock". Mailing: The State Hermitage Museum, 2, Dvortsovaya square, Saint Petersburg, Russia 190000. 